\title{
A comparative recombination analysis of human coronaviruses and implications for the SARS-CoV-2 pandemic
}

Simon Pollett ${ }^{1,2,3}$, Matthew A Conte ${ }^{1}$, Mark Sanborn ${ }^{1}$, Richard G Jarman ${ }^{1}$, Grace M. Lidl ${ }^{1}$, Kayvon Modjarrad $^{4}$, Irina Maljkovic Berry ${ }^{1 *}$

${ }^{1}$ Viral Diseases Branch, Walter Reed Army Institute of Research, MD, USA

${ }^{2}$ Infectious Disease Clinical Research Program, Department of Preventive Medicine and Biostatistics, Uniformed Services University of the Health Sciences, Bethesda, MD, USA

${ }^{3}$ Henry M. Jackson Foundation for the Advancement of Military Medicine, Inc., Bethesda, MD, USA

${ }^{4}$ Emerging Infectious Diseases Branch, Walter Reed Army Institute of Research, MD, USA

*Corresponding author

\begin{abstract}
The SARS-CoV-2 pandemic prompts evaluation of recombination in human coronavirus (hCoV) evolution. We undertook recombination analyses of 158,118 public seasonal hCoV, SARS-CoV-1, SARSCoV-2 and MERS-CoV genome sequences using the RDP4 software. We found moderate evidence for 8 SARS-CoV-2 recombination events, two of which involved the spike gene, and low evidence for one SARS-CoV-1 recombination event. Within MERS-CoV, 229E, OC43, NL63 and HKU1 datasets, we noted 7, $1,9,14$, and 1 high-confidence recombination events, respectively. There was propensity for recombination breakpoints in structural genes, and recombination severely skewed the temporal structure of these data, especially for NL63 and OC43. Bayesian time-scaled analyses on recombinantfree data indicated the sampled diversity of seasonal CoVs emerged in the last 70 years, with $229 \mathrm{E}$ displaying continuous lineage replacements. These findings emphasize the importance of genomic based surveillance to detect recombination in SARS-CoV-2, particularly if recombination may lead to immune evasion.
\end{abstract}

\section{INTRODUCTION}

The emergence of SARS-CoV-2 has generated interest in role of recombination in the evolution of this and other human coronaviruses (hCoV). Recombination has been observed in many RNA viruses and is noted to occur at a higher frequency in positive-sense RNA viruses, a category that includes SARS-CoV-2 and other medically important coronaviruses (1-3). From an evolutionary biology perspective, it remains unclear why recombination occurs in RNA viruses. Hypotheses include recombination being an incidental outcome of RNA polymerase function, or an evolutionary favorable purge of deleterious genotypes and/or generation of advantageous genotypes (1).

While several studies have examined the putative role of recombination in the zoonotic emergence of SARS-CoV-2 (4-6), few have focused on the emergence of recombination during the first year of the SARS-CoV-2 pandemic. To date, none have systematically examined the genetic propensity of recombination across all human coronaviruses in order to predict the possible evolutionary future of 
SARS-CoV-2, despite prior observations of recombination in OC43-hCoV, HKU1-hCoV, NL63-hCoV, and MERS-COV (7-19).

RNA virus recombination has been associated with changes in host range, host response and virulence (1). Identifying the presence of recombination, or predicting the risk of recombination, in viral populations of SARS-CoV-2 is critical for several reasons. First, circulating recombinants may complicate molecular diagnostic performance. Second, recombinants may cause rapid escape from naturally acquired immunity, as has been observed in the norovirus genus, which has caused pandemics due to the rapid emergence of new genotypes generated by recombination of structural genes (20). For SARSCoV-2, such an event may have major implications, especially if circulating recombinant results in escape from both natural and vaccine induced immunity (21). Finally, genomic epidemiology has increasingly been shown to be an important public health tool for SARS-CoV-2 and failing to accommodate for recombinant data may lead to incorrect epidemiological inference because of possible phylogenetic incongruence $(22,23)$.

We undertook a comparative recombination analysis of all published SARS-CoV-1, SARS-CoV-2, MERSCoV and seasonal hCoV (OC43, 229E, NL63 and HKU1) genomes. Specifically, we aimed to identify the frequency and genomic location of recombination events in these hCoV types, and estimate the impact of these recombinants on hCoV emergence dates (TMRCA). As part of this analysis, we reconstructed the time-scale of circulating seasonal hCoV evolution and lineage replacement to provide insights into the possible future evolutionary trajectory of SARS-CoV-2.

\section{RESULTS}

There is moderate evidence for recombination in several SARS-CoV-2 genomes through October 2020 Among SARS-CoV-2 genomes, we detected a total of 8 recombination events detected by at least three detection methods, though these events were possibly caused by another non-recombinant process (Table 1). Some of these events, while not supported by a high level of evidence, were noted in multiple sequences and may therefore represent circulating recombinant forms (Supplemental Table 1). However, these recombination events were not found across all subsampled datasets. For those recombination events with moderate evidence, we noted that half of the events $(4 / 8)$ comprised breakpoints in the structural genes, and one quarter (2/8) occurred within the spike gene (Supplemental Table 2).

Table 1. Frequency of recombination events detected in 229E, NL63, OC43, HKU1, MERS-CoV, SARS-CoV1 and SARS-CoV-2, stratified by level of evidence

\begin{tabular}{|c|c|c|c|c|c|}
\hline $\begin{array}{l}\text { Coronavirus } \\
\text { species }\end{array}$ & $\begin{array}{l}n \text { genome } \\
\text { sequences }\end{array}$ & $\begin{array}{l}\text { Recombination } \\
\text { events detected } \\
\text { by any method }\end{array}$ & $\begin{array}{l}\text { Recombination } \\
\text { events detected } \\
\text { by } \geq 3 \text { methods }\end{array}$ & $\begin{array}{l}\text { Recombination events } \\
\text { detected by } \geq 3 \text { methods } \\
\text { and without another } \\
\text { evolutionary process } \\
\text { possibly explaining the } \\
\text { recombination signal } \\
\text { (high evidence) }\end{array}$ & $\begin{array}{c}\text { Recombination } \\
\text { events with high } \\
\text { level evidence } \\
\text { seen in multiple } \\
\text { genomes }\end{array}$ \\
\hline hCoV-229E & 22 & 4 & 3 & 1 & 1 \\
\hline hCoV-NL63 & 65 & 31 & 24 & 14 & 7 \\
\hline hCoV-OC43 & 138 & 23 & 16 & 9 & 6 \\
\hline
\end{tabular}




\begin{tabular}{lccccc} 
hCoV-HKU1 & 37 & 14 & 9 & 1 & 1 \\
MERS-CoV & 365 & 12 & 10 & 7 & 6 \\
SARS-CoV-1 & 49 & 1 & 0 & 0 & 0 \\
SARS-CoV-2 & $100296^{\mathrm{a}}$ & 33 & 8 & 0 & 0 \\
\hline
\end{tabular}

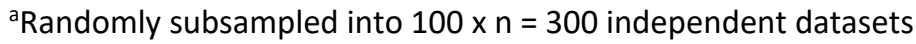

\section{Recombination is relatively frequent in seasonal endemic coronaviruses and has a propensity for the structural genes}

Within the 229E, OC43, NL63 and HKU1 datasets, we noted 1, 9, 14, and 1 high confidence

recombination events, respectively (Table 1 ). These recombination events were found in $13.6 \%, 20.3 \%$, $89.2 \%$, and $35.1 \%$ of the analyzed sequences, respectively. In the OC43 and NL63 datasets, we noted significantly more breakpoints in the structural genes than in the non-structural genes $(p=0.0004, p=$ 0.0032 respectively) (Figure 1 ).

Figure 1. Estimated recombination breakpoint positions NL63, OC43 and MERS-CoV whole genomes. P-values for the frequency of recombination breakpoints in structural versus non-structural genes are derived by the $\chi^{2}$ test. Approximate breakpoints are breakpoints that could not be placed with certainty due to overlapping recombination or other reasons.

Recombinants were noted across entire clades of HKU1, NL63 and OC43 viruses, with clusters of genomes sharing identical recombination patterns, indicating spread of a recombinant virus following its emergence through a recombination event. The OC43 and NL63 datasets also contained genomes with unique recombination patterns (singletons) (Figure 2, Figure 3). Furthermore, we noticed some singletons falling within already recombinant clades, indicating presence of successful second generation recombination (Figure 2, Figure 3). A $10^{\text {th }}$ OC43 event with moderate recombination evidence based on RDP4 results showed topological incongruence in subgenomic phylogenetic trees and was therefore removed for further time-scaled analyses.

Figure 2. Maximum likelihood phylogeny of recombinants in NL63. Scale represents nucleotides per site. Recombinant events with multiple genomes are marked in blue, or as singletons are marked in yellow. Phylogeny was rooted with a $229 E$ outgroup (removed for clarity).

Figure 3. Maximum likelihood phylogenies of recombinants in OC43. Scale represents nucleotides per site. Recombinant events with multiple genomes are marked in blue, or as singletons are marked in yellow. Phylogeny was rooted with an HKU1 outgroup (removed for clarity).

\section{MERS-COV but not SARS-CoV-1 is characterized by frequent recombination with a propensity for structural genes}

Within the MERS-CoV dataset, we detected 7 recombination events with a high level of confidence (detected by 3 or more methods and not explained by another evolutionary process) (Table 1). These recombination events were found in 14.5\% of all analyzed MERS-CoV genomes. Of these, 6 were found across clades, suggesting recombinants were sufficiently fit for onward transmission (Figure 4). We noted recombinant clades defined by camel hosts, as well as camel and human hosts (Figure 4), suggestive of inter-host recombinant spread. Moreover, we noted significantly more breakpoints in the structural genes compared to the non-structural genes $(p<0.001)$ (Figure 1$)$. We noted only a single low 
evidence recombination event involving the structural region of the SARS-CoV1 genome (4503 -25998 $\mathrm{nt}$ ), but this was not confirmed by multiple methods (Table 1).

Figure 4. Maximum likelihood phylogeny of recombinants in MERS-CoV. Scale represents nucleotides per site. A) Taxa colored by host (camel = black, human = green). B) Colored taxa indicate confirmed recombinant clades.

\section{Recombination in seasonal coronaviruses substantially alters estimates of temporal structure}

Our approach to identification of recombinants and their subsequent removal from the datasets led to major improvements in estimated temporal structure of hCoV-OC43, hCoV-NL63 and hCoV-HKU1. These changes involved both the regression coefficient and the regression intercept, which serve as crude estimates of evolutionary rates and TMRCA, and are often used to assess the clock-likeness (linear relationship of genetic distance across sequence sampling times) of the data for further analyses (Table 2). In contrast, removal of the single $229 \mathrm{E}$ recombinant did not cause substantive change in estimated temporal structure as estimated by regression coefficient and TMRCA (Table 2).

Table 2. Root-to-tip regression coefficient and intercept of seasonal hCoV phylogenies with and without recombinants removed

\begin{tabular}{lcccc}
\hline \multicolumn{5}{c}{ Recombinants not removed } \\
\hline Lineage & Sequences ( $\mathrm{n}$ ) & Date range (years) & Slope coefficient ${ }^{\mathrm{a}}$ & Intercept (TMRCA) $^{\mathrm{b}}$ \\
\hline hCoV-229E & 22 & 26.36 & $2.69 \times 10^{-4}$ & 1990 A.D \\
hCoV-NL63 & 65 & 35.38 & $-1.00 \times 10^{-4}$ & 2149 A.D \\
hCoV-OC43 & 138 & 33.98 & -0.00 & 27359 A.D \\
hCoV-HKU1 & 37 & 14.17 & $4.52 \times 10^{-4}$ & 1941 A.D \\
\hline \multicolumn{5}{c}{ Recombinants removed } \\
\hline Lineage & Sequences (n) & Date range (years) & Slope coefficient ${ }^{\text {a }}$ & Intercept $^{\text {b }}$ \\
\hline hCoV-229E & 19 & 26.36 & $2.65 \times 10^{-4}$ & 1990 A.D \\
hCoV-NL63* & 56 & 35.04 & $7.68 \times 10^{-5}$ & 1944 A.D \\
hCoV-OC43 & 110 & 34.00 & $2.83 \times 10^{-4}$ & 1967 A.D \\
hCoV-HKU1 & 24 & 13.41 & $1.00 \times 10^{-3}$ & 1978 A.D \\
\hline
\end{tabular}

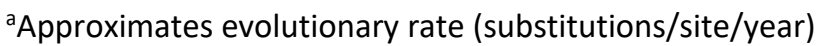

${ }^{\mathrm{b}}$ Approximates TMRCA

*Non-recombinant region was used, with genomes containing breakpoints in this region removed $(\mathrm{N}=9)$

\section{The current global diversity of seasonal hCoVs arose across the last 70 years}

We estimated a TMRCA date (year A.D) of 1989, 1970, 1964, and 1951 for the 229E, OC43, NL63 and HKU1 lineages, respectively, indicating relatively recent emergence of the current seasonal hCoV lineages (Table 3, Supplemental Figures 1-4). Importantly, these do not necessarily represent de novo emergence of these viruses from animal origins but rather may represent the divergence from older OC43, NL63 and HKU1 lineages, respectively. Indeed, the TMRCA for 229E is preceded by historical descriptions of the circulation of this virus in humans, which was discovered in 1965 (24). We therefore extended our 229E full genome analysis and inferred TMRCA estimates from partial genome datasets of the complete $N$ gene $(N=102$, length=1167nt), the complete $S$ gene (Supplemental Figure 5$)(N=78$, 
length=3522nt), the RBD (S1) domain S gene ( $N=90$, length=1650nt), and concatenated $\mathrm{S}$ and $\mathrm{N}$ genes ( $N=64$, length=4689nt). These yielded TMRCA point estimates between 1964 and 1969 (229E RBD S1 gene TMRCA $=1965.6 ; 229 \mathrm{E}$ gene TMRCA $=1964.8 ; 229 \mathrm{E} \mathrm{N}$ gene TMRCA $=1969.1 ; 229 \mathrm{E} \mathrm{N}$ and $\mathrm{S}$ gene TMRCA = 1964.0).

Table 3. Bayesian TMRCA estimates for 229E, HKU1, NL63 and OC43 ${ }^{a}$

\begin{tabular}{lcccccc} 
& $\begin{array}{c}\text { TMRCA } \\
\text { (A.D) }\end{array}$ & $\begin{array}{c}\text { Lower } \\
95 \% \text { HPD }\end{array}$ & $\begin{array}{c}\text { Upper } \\
95 \% \text { HPD }\end{array}$ & $\begin{array}{c}\text { Nucleotide } \\
\text { Subst Model }\end{array}$ & Clock model & $\begin{array}{c}\text { Demographic } \\
\text { model }\end{array}$ \\
\hline 229E $^{\text {a }}$ & 1989 & 1988 & 1990 & SYM+l+G & Strict & Constant \\
HKU1 $^{\text {a }}$ & 1951 & 1842 & 1998 & GTR+G+I & UCLN & Bayesian Skyline \\
NL63 $^{\text {b }}$ & 1964 & 1945 & 1978 & HKY+I & UCLN & Bayesian Skyline \\
OC43 $^{\text {a }}$ & 1970 & 1960 & 1978 & GTR+I & UCLN & Bayesian Skyline \\
\hline
\end{tabular}

${ }^{\mathrm{a}}$ Recombinant genomes removed

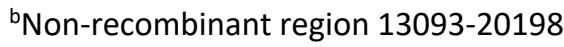

UCLN = uncorrelated lognormal; TMRCA = time to most recent common ancestor

\section{DISCUSSION}

We performed a comprehensive recombination analysis across all medically important human coronaviruses, with an overarching aim to identify the current and future risk of recombinant emergence in the SARS-CoV-2 pandemic. We note moderate evidence for SARS-CoV-2 recombination during the first year of the COVID-19 pandemic. In other hCoV species, we note that recombination has a predilection for structural genes and is relatively frequent in most medically significant hCoV over a relatively short evolution timescale. These findings are timely with the announcement of a more recent unpublished recombinant SARS-CoV-2 strain (25), as well as the recent focus on how insights can be learned from the functional implications of antigenic evolution now demonstrated in seasonal coronaviruses (26).

From more than 100,000 SARS-CoV-2 genomes, we noted 8 instances of recombination with moderate confidence. However, all were flagged by the RDP4 software as being possibly driven by other processes despite support by three or more recombination detection methods. This may reflect the relatively lower viral diversity across the first year of the pandemic. Similarly, we did not detect a high-confidence recombination signal in SARS-CoV-1, a virus with a limited temporal distribution. In contrast, we show that recombination was relatively frequent in seasonal coronavirus and MERS-CoV datasets comprising a longer period of sampling, including recombinants sufficiently fit for onward transmission. Furthermore, our analyses show that three of the coronaviruses (MERS, OC43 and NL63) had breakpoint propensity for structural genes. Several reasons for this may exist, such as inherent structural similarities of the coronaviruses in this region causing enhanced enzyme slippage, or positive selection pressure. The latter may be correlated with evasion of the human immune system.

Our endemic coronavirus analyses also highlighted that recombination affected the estimated temporal structure of coronavirus sequence datasets. This serves as a caution for genomic epidemiology studies which do not identify and account for recombinants in SARS-CoV-2 and other coronavirus analyses. Indeed, recombination has long been known to be a cause of phylogenetic incongruence for other viruses (23). Our time-scaled evolutionary analyses, adjusted for recombination to restore a molecular 
clock signal, yielded insights into the recent epidemiology of seasonal coronaviruses. We noted that the current sampled diversity of seasonal coronaviruses has emerged within a 70 year period, punctuated by new lineage emergence at intervals ranging from 5 to 20 years. Caution is required in inferring that these viruses spilled over into humans at these timepoints, however, as this may reflect the divergence of new lineages from prior, unsampled older viral populations circulating in humans. In the case of $229 \mathrm{E}$ $\mathrm{hCoV}$, we noted that the full genome TMRCA estimates were preceded by the clinical reports of $229 \mathrm{E}$ infection in the 1960s (24) and a spike gene analysis incorporating older partial genome sequences from the earlier $20^{\text {th }}$ century showed lineage extinction and replacement. This is consistent with previous analyses suggesting that $229 \mathrm{E}$ evolution is characterized by prior lineage extinction and new lineage emergence (27).

It is important to note that our RNA genomic recombination detection in sequence data remains a statistical estimation only, as previously discussed in detail for dengue viruses (28). In addition, the size of our SARS-CoV-2 dataset was computationally prohibitive to perform recombination detection across all data, which might have resulted in missing additional recombination events. Also, unsampled data are a pervasive technical risk for recombination detection, as demonstrated by the variable finding of recombinant events across subsampled SARS-CoV2 data. Finally, our strict criteria for identifying recombination events with high confidence may have resulted in the removal of some true recombinant events.

Still, these findings provide critical insights into the possible projected evolution of SARS-CoV2, as it becomes an endemic viral pathogen in human populations. Recombination in other RNA viruses has been associated with changes in host tropism, virulence or epidemiology (1). Ongoing genomic-based COVID-19 surveillance has recently been highlighted as a critical public health tool to detect novel SARSCoV-2 variants, such as the B.1.1.7, B.1.351, and P.1 variants $(29,30)$. Robust genomic surveillance will be essential for the timely detection of recombination in SARS-CoV-2, which may have implications for the diagnosis of and immunity to this pandemic pathogen.

\section{MATERIALS AND METHODS}

\section{Data curation and alignment}

Full genomes of endemic seasonal hCoVs, MERS-CoV and SARS-CoV-1 were downloaded from the NIAID Virus Pathogen Database and Analysis Resource (ViPR) (31, 32). Specifically, we obtained $n=22 \mathrm{hCoV}$ 229E whole genomes, $n=138 \mathrm{hCoV-OC43}$ whole genomes, $n=68$ hCoV-NL63 whole genomes, $n=37$ HKU1 whole genomes, $n=365$ MERS-CoV whole genomes (including human and camel host), and $n=49$ SARS-CoV-1 whole genomes. We excluded laboratory constructs and sequences without host, collection date and location history. Datasets were aligned using MAFFT (33), with manual alignment thereafter in MEGA v6.0 (34). Alignment lengths were adjusted such that all genomes from one alignment were of approximately equal length (OC43 $=30,639 \mathrm{nt}$; NL63 = 27,483 nt; HKU1 = 29,892 nt; 229e = 27,292 nt; MERS $=29,985 \mathrm{nt}$; SARS1 $=29,719 \mathrm{nt}$ ). Partial genomes were excluded. Accession numbers for these data are presented in Supplemental Table 3.

All available full SARS-CoV-2 genomes ( $n=157,439)$ up to October 23, 2020 were downloaded from the GISAID database (35). These data were curated by removing (i) any partial genomes ( $<90 \%$ full genome length), (ii) any genomes with $>100$ continuous ambiguous base calls (Ns), and (iii) removing bat and pangolin non-human genomes. A single genome (Italy/CAM-IZSM-45946/2020) was removed due to the 
presence of non-IUPAC nucleotide codes. The remaining 100,296 genomes were aligned to the MN908947.3 reference using mafftc (7.471) in the MAFFT software (33). Alignment ends (first 265 and last $229 \mathrm{nt}$ ) were trimmed using the trimal command (36). This yielded a final alignment of length $29,409 \mathrm{bp}$.

\section{Recombination detection and determination of breakpoints}

Datasets underwent recombination detection using the RDP4 software (37). Recombination signal detection was performed with a suite of methods: the original RDP method (38), BOOTSCAN (39), MAXCHI (40), CHIMAERA (41), 3SEQ (42), GENECONV (43), SISCAN (44). Following the detection of a 'recombination signal' with these methods, the approximate breakpoint positions were determined using a hidden Markov model, BURT, and the recombinant sequence identified using the PHYLPRO (45), VISRD (46), and EEEP methods (47).

For SARS-CoV2, due to the prohibitive computational demand of recombination detection analysis in a dataset of this size, we randomly subsampled, with replacement, $\mathrm{n}=300$ sequences with $100 \mathrm{x}$ iterations ( $n=30,000$ full genomes) to perform recombination detection.

As individual recombination detection methods may have limited specificity, we developed a customized framework to ascertain the level of evidence for those recombination events detected in these datasets. Recombination events identified by only one or two methods in RDP4 were assigned a 'low' level of confidence, and those identified by at least three methods in RDP4 were assigned at least a 'moderate' level of confidence. We further assigned a 'high' level of confidence for those recombination events identified by at least three RDP4 methods with no other identified process which may have explained the recombination signal (37). The evolutionary processes that might be misinterpreted as recombination are typically caused by a combination of mutation rate variation between sites superimposed on mutation rate variation along lineages ( $M$. Darren, personal communication, Dec 4, 2020). For those recombination events with a high level of evidence, the location of breakpoints were plotted across the whole genome and a $\chi^{2}$ test used to compare frequency of breakpoints at structural versus non-structural regions. Breakpoints that could not be placed with certainty due to overlapping recombination or other reasons were not included in the analyses.

\section{Estimation of temporal structure of hCoV with and without recombinant strains}

Datasets for each hCoV species underwent nucleotide model substitution selection using JModelTest2 (48), with model selection as follows: $229 \mathrm{E}=\mathrm{SYM}+\mathrm{I}+\mathrm{G}, \mathrm{HKU1}=\mathrm{GTR}+\mathrm{G}+\mathrm{I}, \mathrm{NL63}=\mathrm{GTR}+\mathrm{I}, \mathrm{OC43}=$ $\mathrm{GTR}+\mathrm{G}+\mathrm{I}$. A maximum likelihood phylogeny was inferred using the PhyML software (49), with aLRT for node support and tips labeled by date of collection. Root-to-tip regression was performed using the Temp-Est tool (50), with slope coefficient and intercept values used as preliminary estimates of evolutionary rate and time-to-most-common recent ancestor (TMRCA), respectively. Confirmed recombinant sequences were annotated on these phylogenies to identify recombinant clades and singletons. Phylogenetic analyses were repeated with the recombinants removed to estimate the impact of recombination on time-scales and temporal structure of hCoV evolution. For NL63, removal of recombinant genomes resulted in a small dataset and a small remaining fraction of the initial phylogenetic tree. Therefore, NL63 genomes were screened for a common region without any recombination signal. A region of 7105 nts (region 13093-20198 of the alignment) was found in which most genomes $(n=56)$ had no recombination signal, and this region was used for subsequent time-scaled 
phylogenetic analyses, with the 9 genomes with recombination breakpoints in this region removed from the dataset.

\section{Estimating time-scales of emergence of the currently circulating seasonal hCoV lineages}

We leveraged recombination-free endemic seasonal hCoV datasets, in addition to removing other rootto-tip regression outlier genomes $(n=3+4$ identical genomes for 0 C43, $n=1$ for NL63, $n=0$ for $229 e, n$ $=2$ for HKU1), to reconstruct the time-scale of emergence of currently circulating seasonal hCoV lineages across the 229E, OC43, NL63 and HKU1 types. We focused on these coronaviruses because they are well established viruses within human populations and may serve as a model for the projected evolutionary future of SARS-CoV-2 which is now already an endemic RNA virus. This is in contrast to the now extinct SARS-CoV-1 virus, and MERS-CoV, the latter which continues to be defined by more sporadic and discrete spillover events (51).

Time-scaled genealogies of these viruses were inferred using the BEAST software 1.8.4 (52). To minimize the risk of model misspecification, we inferred maximum clade credibility phylogenies with combinations of demographic models (constant, exponential and skyline population models) and molecular clock models (strict versus relaxed) (Supplemental Table 4). For each hCoV dataset, the optimal combination of demographic and molecular clock model was selected by logarithmic marginal likelihoods inferred by the path-sampling/ stepping-stone method (47).

\section{DISCLAIMER}

Material has been reviewed by the Walter Reed Army Institute of Research. There is no objection to its presentation and/or publication. The View(s) expressed are those of the authors and do not necessarily reflect the official views of the Uniformed Services University of the Health Sciences, Henry M. Jackson Foundation for the Advancement of Military Medicine, Inc., Department of Health and Human Services, the National Institutes of Health, the Departments of the Army, Navy or Air Force, the Department of Defense, or the U.S. Government.

\section{ACKNOWLEDGEMENTS}

The authors would like to acknowledge all the authors and originating and submitting laboratories of the sequences from GISAID's EpiCov that were used in our analyses. A full acknowledgments list is submitted as Supplemental Table 5. This study was funded by the Global Emerging Infections Surveillance (GEIS) Branch (ProMIS ID: P0140_20_WR) and the US Department of Defense Health Agency. Support for this work was provided by the Infectious Disease Clinical Research Program (IDCRP), a Department of Defense program executed through the Uniformed Services University of the Health Sciences, Department of Preventive Medicine and Biostatistics through a cooperative agreement with The Henry M. Jackson Foundation for the Advancement of Military Medicine, Inc. (HJF).

\section{COMPETING INTERESTS}

The authors do not have any competing interests.

\section{REFERENCES}


bioRxiv preprint doi: https://doi.org/10.1101/2021.03.07.434287; this version posted March 8, 2021. The copyright holder for this preprint

1. Simon-Loriere E, Holmes EC. Why do RNA viruses recombine? Nat Rev Microbiol. 2011;9(8):61726.

2. Dhama K, Khan S, Tiwari R, Sircar S, Bhat S, Malik YS, et al. Coronavirus Disease 2019-COVID-19. Clin Microbiol Rev. 2020;33(4).

3. Cheng VC, Lau SK, Woo PC, Yuen KY. Severe acute respiratory syndrome coronavirus as an agent of emerging and reemerging infection. Clin Microbiol Rev. 2007;20(4):660-94.

4. Neches RY, McGee MD, Kyrpides NC. Recombination should not be an afterthought. Nat Rev Microbiol. 2020;18(11):606.

5. Paraskevis D, Kostaki EG, Magiorkinis G, Panayiotakopoulos G, Sourvinos G, Tsiodras S. Fullgenome evolutionary analysis of the novel corona virus (2019-nCoV) rejects the hypothesis of emergence as a result of a recent recombination event. Infect Genet Evol. 2020;79:104212.

6. Wu A, Niu P, Wang L, Zhou H, Zhao X, Wang W, et al. Mutations, Recombination and Insertion in the Evolution of 2019-nCoV. bioRxiv. 2020.

7. Vijgen L, Keyaerts E, Moës E, Thoelen I, Wollants E, Lemey P, et al. Complete genomic sequence of human coronavirus OC43: molecular clock analysis suggests a relatively recent zoonotic coronavirus transmission event. J Virol. 2005;79(3):1595-604.

8. Zhang Y, Li J, Xiao Y, Zhang J, Wang Y, Chen L, et al. Genotype shift in human coronavirus OC43 and emergence of a novel genotype by natural recombination. J Infect. 2015;70(6):641-50.

9. Zhang Z, Shen L, Gu X. Evolutionary Dynamics of MERS-CoV: Potential Recombination, Positive Selection and Transmission. Sci Rep. 2016;6:25049.

10. Zhang XW, Yap YL, Danchin A. Testing the hypothesis of a recombinant origin of the SARSassociated coronavirus. Arch Virol. 2005;150(1):1-20.

11. Liu P, Shi L, Zhang W, He J, Liu C, Zhao C, et al. Prevalence and genetic diversity analysis of human coronaviruses among cross-border children. Virol J. 2017;14(1):230.

12. Kin N, Miszczak F, Lin W, Gouilh MA, Vabret A. Genomic Analysis of 15 Human Coronaviruses OC43 (HCoV-OC43s) Circulating in France from 2001 to 2013 Reveals a High Intra-Specific Diversity with New Recombinant Genotypes. Viruses. 2015;7(5):2358-77.

13. Lau SK, Feng Y, Chen H, Luk HK, Yang WH, Li KS, et al. Severe Acute Respiratory Syndrome (SARS) Coronavirus ORF8 Protein Is Acquired from SARS-Related Coronavirus from Greater Horseshoe Bats through Recombination. J Virol. 2015;89(20):10532-47.

14. Lau SK, Lee P, Tsang AK, Yip CC, Tse H, Lee RA, et al. Molecular epidemiology of human coronavirus OC43 reveals evolution of different genotypes over time and recent emergence of a novel genotype due to natural recombination. J Virol. 2011;85(21):11325-37.

15. Dominguez SR, Sims GE, Wentworth DE, Halpin RA, Robinson CC, Town CD, et al. Genomic analysis of 16 Colorado human NL63 coronaviruses identifies a new genotype, high sequence diversity in the N-terminal domain of the spike gene and evidence of recombination. J Gen Virol. 2012;93(Pt 11):2387-98.

16. Pyrc K, Dijkman R, Deng L, Jebbink MF, Ross HA, Berkhout B, et al. Mosaic structure of human coronavirus NL63, one thousand years of evolution. J Mol Biol. 2006;364(5):964-73.

17. Woo PC, Lau SK, Yip CC, Huang Y, Tsoi HW, Chan KH, et al. Comparative analysis of 22 coronavirus HKU1 genomes reveals a novel genotype and evidence of natural recombination in coronavirus HKU1. J Virol. 2006;80(14):7136-45.

18. Sabir JS, Lam TT, Ahmed MM, Li L, Shen Y, Abo-Aba SE, et al. Co-circulation of three camel coronavirus species and recombination of MERS-CoVs in Saudi Arabia. Science. 2016;351(6268):81-4.

19. Wang Y, Liu D, Shi W, Lu R, Wang W, Zhao Y, et al. Origin and Possible Genetic Recombination of the Middle East Respiratory Syndrome Coronavirus from the First Imported Case in China: Phylogenetics and Coalescence Analysis. mBio. 2015;6(5):e01280-15. 
bioRxiv preprint doi: https://doi.org/10.1101/2021.03.07.434287; this version posted March 8, 2021. The copyright holder for this preprint

20. Eden JS, Tanaka MM, Boni MF, Rawlinson WD, White PA. Recombination within the pandemic norovirus GII.4 lineage. J Virol. 2013;87(11):6270-82.

21. Dearlove B, Lewitus E, Bai H, Li Y, Reeves DB, Joyce MG, et al. A SARS-CoV-2 vaccine candidate would likely match all currently circulating variants. Proc Natl Acad Sci U S A. 2020;117(38):23652-62.

22. https://nextstrain.org/ncov/global, accessed December 31, 2020.

23. Pérez-Losada M, Arenas M, Galán JC, Palero F, González-Candelas F. Recombination in viruses: mechanisms, methods of study, and evolutionary consequences. Infect Genet Evol. 2015;30:296-307.

24. Tyrrell DA, Bynoe ML. Cultivation of viruses from a high proportion of patients with colds. Lancet. 1966;1(7428):76-7.

25. https://www.newscientist.com/article/2268379-two-coronavirus-variants-have-merged-hereswhat-you-need-to-know/, cited Feb 202021.

26. https://www.biorxiv.org/content/10.1101/2020.12.17.423313v1.full.pdf, accessed Dec 312020.

27. Kahn JS, McIntosh K. History and recent advances in coronavirus discovery. Pediatr Infect Dis J. 2005;24(11 Suppl):S223-7, discussion S6.

28. Chen R, Vasilakis N. Dengue--quo tu et quo vadis? Viruses. 2011;3(9):1562-608.

29. https://virological.org/t/preliminary-genomic-characterisation-of-an-emergent-sars-cov-2-

lineage-in-the-uk-defined-by-a-novel-set-of-spike-mutations/563, accessed Dec 312020.

30. https://bv-brc.org/view/VariantLineage/\#view_tab=overview accessed Mar 042021.

31. www.viprbrc.org, latest access Apr 072020.

32. Pickett BE, Sadat EL, Zhang Y, Noronha JM, Squires RB, Hunt V, et al. ViPR: an open bioinformatics database and analysis resource for virology research. Nucleic Acids Res. 2012;40(Database issue):D593-8.

33. Katoh K, Standley DM. MAFFT: iterative refinement and additional methods. Methods Mol Biol. 2014;1079:131-46.

34. Tamura K, Stecher G, Peterson D, Filipski A, Kumar S. MEGA6: Molecular Evolutionary Genetics Analysis version 6.0. Mol Biol Evol. 2013;30(12):2725-9.

35. https://www.gisaid.org/, accessed Oct 232020.

36. Capella-Gutiérrez S, Silla-Martínez JM, Gabaldón T. trimAl: a tool for automated alignment trimming in large-scale phylogenetic analyses. Bioinformatics. 2009;25(15):1972-3.

37. Martin DP, Murrell B, Golden M, Khoosal A, Muhire B. RDP4: Detection and analysis of recombination patterns in virus genomes. Virus Evol. 2015;1(1):vev003.

38. Martin D, Rybicki E. RDP: detection of recombination amongst aligned sequences.

Bioinformatics. 2000;16(6):562-3.

39. Martin DP, Posada D, Crandall KA, Williamson C. A modified bootscan algorithm for automated identification of recombinant sequences and recombination breakpoints. AIDS Res Hum Retroviruses. 2005;21(1):98-102.

40. Smith JM. Analyzing the mosaic structure of genes. J Mol Evol. 1992;34(2):126-9.

41. Posada D, Crandall KA. Evaluation of methods for detecting recombination from DNA sequences: computer simulations. Proc Natl Acad Sci U S A. 2001;98(24):13757-62.

42. Lam HM, Ratmann O, Boni MF. Improved Algorithmic Complexity for the 3SEQ Recombination Detection Algorithm. Mol Biol Evol. 2018;35(1):247-51.

43. Padidam M, Sawyer S, Fauquet CM. Possible emergence of new geminiviruses by frequent recombination. Virology. 1999;265(2):218-25.

44. Gibbs MJ, Armstrong JS, Gibbs AJ. Sister-scanning: a Monte Carlo procedure for assessing signals in recombinant sequences. Bioinformatics. 2000;16(7):573-82.

45. Weiller GF. Phylogenetic profiles: a graphical method for detecting genetic recombinations in homologous sequences. Mol Biol Evol. 1998;15(3):326-35. 
46. Lemey $\mathrm{P}$, Lott $\mathrm{M}$, Martin DP, Moulton V. Identifying recombinants in human and primate immunodeficiency virus sequence alignments using quartet scanning. BMC Bioinformatics. 2009;10:126.

47. Baele G, Li WL, Drummond AJ, Suchard MA, Lemey P. Accurate model selection of relaxed molecular clocks in bayesian phylogenetics. Mol Biol Evol. 2013;30(2):239-43.

48. Darriba D, Taboada GL, Doallo R, Posada D. jModelTest 2: more models, new heuristics and parallel computing. Nat Methods. 2012;9(8):772.

49. Guindon S, Gascuel O. A simple, fast, and accurate algorithm to estimate large phylogenies by maximum likelihood. Syst Biol. 2003;52(5):696-704.

50. Rambaut A, Lam TT, Max Carvalho L, Pybus OG. Exploring the temporal structure of heterochronous sequences using TempEst (formerly Path-O-Gen). Virus Evol. 2016;2(1):vew007.

51. https://apps.who.int/iris/bitstream/handle/10665/326126/WHO-MERS-RA-19.1-eng.pdf, accessed Dec 312020.

52. Suchard MA, Lemey P, Baele G, Ayres DL, Drummond AJ, Rambaut A. Bayesian phylogenetic and phylodynamic data integration using BEAST 1.10. Virus Evol. 2018;4(1):vey016. 


\section{Breakpoints}

- Approximate breakpoints

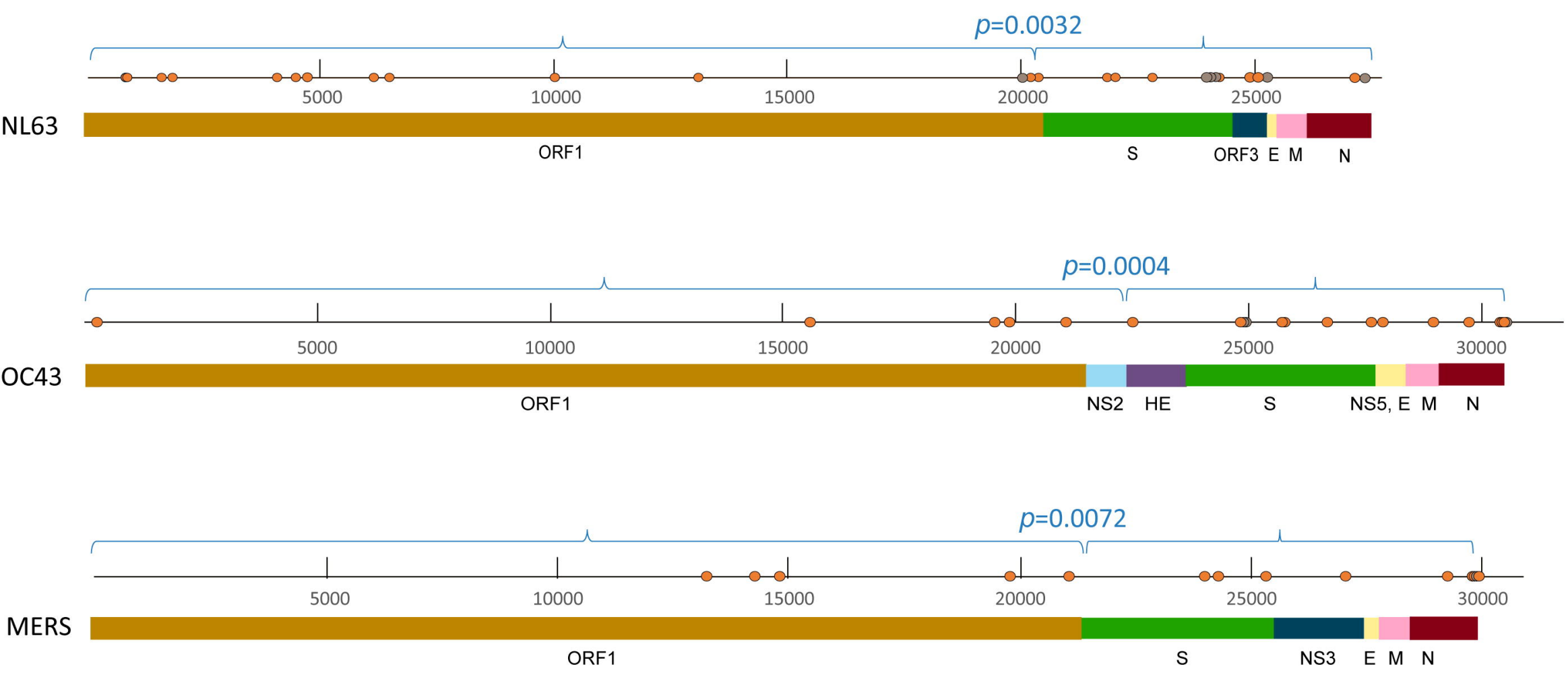


Human_coronavirus_NL63|HCoV_NL63/Haiti__1/2015|KT266906|Haiti|2015_01_16

Human_coronavirus_NL63|CBJ123|JX524171|China|2009_01_24

Human_coronavirus_NL63|NL63/DEN/2009/31|JQ900257|USA|2009_02_21

Human_coronavirus_NL63|HCoV_NL63/Seattle/USA/SC0768/2019|MN306040|USA|2019

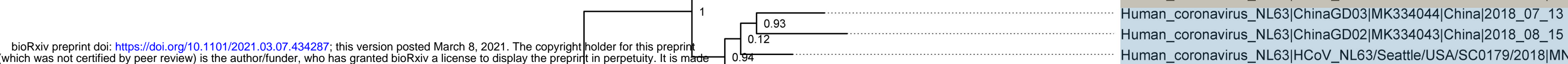

Human_coronavirus_NL63|HCoV_NL63/Seattle/USA/SC0179/2018|MN306018|USA|2018 Human_coronavirus_NL63|ChinaGD05|MK334045|China|2018_08_14

Human_coronavirus_NL63|N07_324B_182X|KY554970|USA|2016

Human_coronavirus_NL63|N07_185B|KY554968|USA|2016

Human_coronavirus_NL63|N07_6B|KY674915|USA|2016

Human_coronavirus_NL63|N06_1144B|KY554967|USA|2016

Human_coronavirus_NL63|N06_1144B|KY554967|USA|2016

Human_coronavirus_NL63|N07_64B|KY674916|USA|2016
Human_coronavirus_NL63|NL63/DEN/2008/16|JQ765566|USA|2008_01_08

Human_coronavirus_NL63|NL63/DEN/2009/6|JQ900255|USA|2009_02_25

Human_coronavirus_NL63|NL63/UF_1/2015|KT381875|USA|2015

Human_coronavirus_NL63|NL63/UF_2/2015|KX179500|USA|2015_09

Human_coronavirus_NL63|NL63/UF_2/2015|KU521535|USA|2015_09 01

Human_coronavirus_NL63|NL63/DEN/2009/20|JQ765567|USA|2009_03_12

Human_coronavirus_NL63|UNKNOWN_DJ009246|DJ009246|Netherlands|2003_01

Human_coronavirus_NL63|UNKNOWN_CS124012|CS124012|Netherlands|2003_01

Human_coronavirus_NL63|Amsterdam_||NC_005831|Netherlands|2003_01

Human_coronavirus_NL63|Amsterdam_||AY567487|Netherlands|2003_01

Human_coronavirus_NL63|NL63/RPTEC/2004|JX504050|USA|2004

Human_coronavirus_NL63|NL63/DEN/2005/1876|JQ765575|USA|2005_11_21

Human_coronavirus_NL63|NL63/DEN/2005/449|JQ900259|USA|2005_02_09

Human_coronavirus_NL63INL63/DEN/2005/1062|JQ765573|USA|2005_04_12

(1)

Human_coronaviru__L63/NL63/DEN/2005/103/JQ765568|USA|2005_01_11

Human_coronavirus_NL63|NL63/DEN/2005/271|JQ765571|USA|2005_01_23

Human_coronavirus_NL63|NL63/DEN/2005/347|JQ765572|USA|2005_02_01

Human_coronavirus_NL63|NL63/human/USA/903_28/1990|KF530109|USA|1990_03_2 Human_coronavirus_NL63|NL63/human/USA/911_56/1991|KF530107|USA|1991_01_24 Human_coronavirus_NL63|NL63/human/USA/905_25/1990|KF530113|USA|1990_05_29 Human_coronavirus_NL63|NL63/human/USA/891_6/1989|KF530108|USA|1989_01_05 Human_coronavirus_NL63|NL63/human/USA/891_4/1989|KF530114|USA|1989_01 03

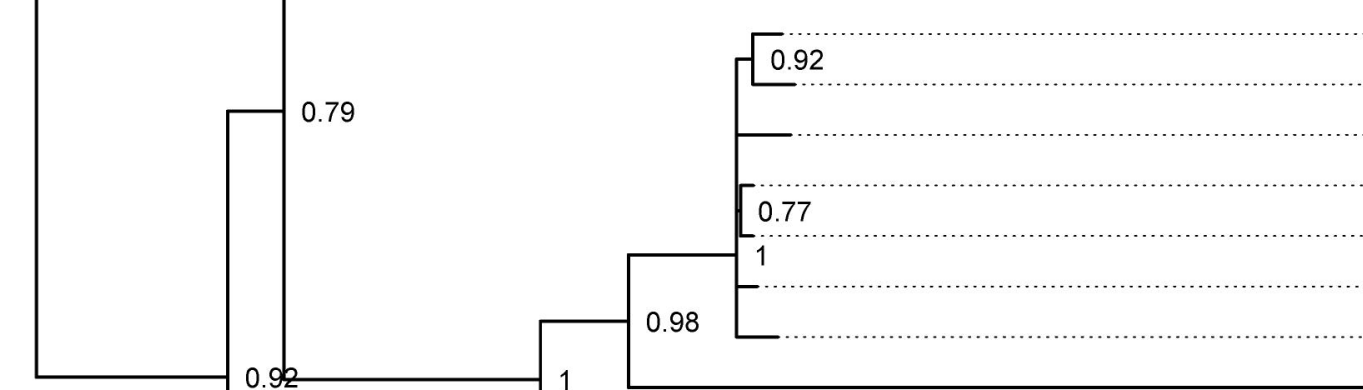

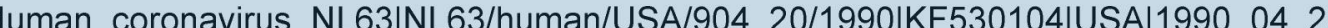
Human_coronavirus_NL63|NL63/human/USA/901_24/1990|KF530111|USA|1990_01_05 Human_coronavirus_NL63|NL63/human/USA/8712_17/1987|KF530106|USA|1987_12_16 Human_coronavirus_NL63|NL63/human/USA/838_9/1983|KF530110|USA|1983_08_16 Human_coronavirus_NL63|N07_262B|KY829118|USA|2015 Human_coronavirus_NL63|N07_196B|KY554969|USA|2016 Human_coronavirus_NL63|N07_468B_176X|KY554971|USA|2016

Human_coronavirus_NL63|NL63/DEN/2005/1862|JQ765574|USA|2005_11

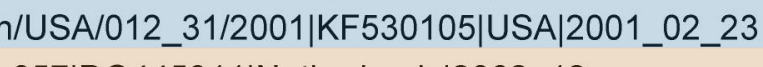
Human_coronavirus_NL63|Amsterdam_057|DQ445911|Netherlands|2002_12

Human_coronavirus_NL63|HCoV_NL63/Seattle/USA/SC2940/2015|KY983586|USA|2015 Human_coronavirus_NL63|CN0601/14|MG772808|South_Korea|2014_11_25 Human_coronavirus_NL63|NL63/DEN/2005/232|JQ765569|USA|2005_01_18 Human_coronavirus_NL63|NL63/DEN/2009/15|JQ765565|USA|2009_02_13 Human_coronavirus_NL63|NL63/DEN/2009/14|JQ765564|USA|2009_03_01 Human_coronavirus_NL63|Amsterdam_496|DQ445912|Netherlands|2003_02 Human coronavirus NL63|NL63/DEN/2005/235|JQ765570|USA|2005 01 Human_coronavirus_NL63|NL63/DEN/2009/9|JQ765563|USA|2009_03_16

Human_coronavirus_NL63|NL63/human/USA/0111_25/2001|KF530112|USA|2001_11_21 .. Human_coronavirus_NL63|NL63/human/USA/0111_25/2001|KF530112|USA|2001_11_ Human_coronavirus_NL63|UNKNOWN_AY518894|AY518894|Nether| Human_coronavirus_NL63|ChinaGD01|MK334046|China|2018_09_01 


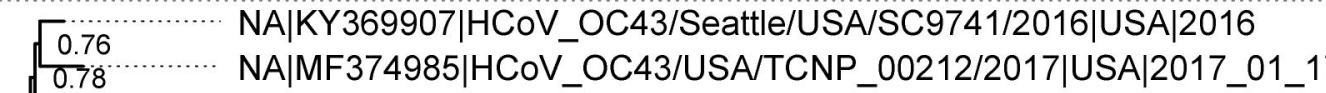
NA|MH121121/HCoV_OC43/USA/ACRI_0213/2016|USA|2016 12 19 NA|KY369906|HCoV_OC43/Seattle/USA/SC622/2016|USA|2016 NA|KY983588|HCoV_OC43/Seattle/USA/SC3118/2015|USA|2015 NA|KY369905|HCoV_OC43/Seattle/USA/SC831/2016|USA|2016 NA|KY967359|HCoV_OC43/Seattle/USA/SC2730/2015/USA/2015 NA|KY967361/HCOVOCA3/SAtI/USA/SC2345/2015 USA/2015 NA|MF374984|HCOV OC43/USA/TCNP 00204/2017/USA|2017 0103 NA|KY967358|HCoV_OC43/Seattle/USA/SC2770/2015|USA|2015 NA|KY684759|HCoV_OC43/Seattle/USA/SC2269/2016|USA|2016 NA|KY983583|HCoV OC43/Seattle/USA/SC2481/2015|USA|2015

0.74 NA|MN310478|HCoV_OC43/Seattle/USA/SC0776/2019|USA|2019 NA|MN306053|HCoV_OC43/Seattle/USA/SC9430/2018|USA|2019 NA|MN306042/HCoV_OC43/Seattle/USA/SC0839/2019|USA|2019 NAIMN306036/HCoV_OC43/Seattle/USAISC0682/2019|USA|2019

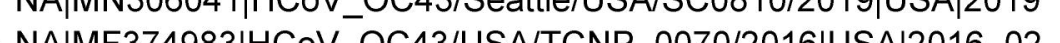
NAKX5389721MY -7774121M NA|KX538966|MY U236/12/Malaysial2012 04 NA|KX538976|MY U1057/12|Malaysia|2012 08 NA|KF923904|12694/2012|China|2012_05

NA|KX538964|MY_U002/12|Malaysia|2012_02_22 NA|MG977452|TNP_12643|Cote_dlvoire|2016_12_10 NA|MG977451|TNP_12636|Cote_dlvoire|2016_12_10 NA|MN026164|OC43_KLF_01_2018|Kenya|2018_01_18 NA|KX538970|MY_U710/12|Malaysia|2012_06_20 NA|KY967356|HCoV OC43/Seattle/USA/SC2924/2015|USA|2015 NA|KX538979|MY U1975/13|Malaysia|2013_02 15 NA|KF923902|12689/2012|China|2012_05 NA|KX538971|MY U732/12|Malaysial2012 06 NA|KX538974|MY_U945/12|Malaysia|2012_08_01 NA|KX538973|MY_U868/12|Malaysia|2012_07_16 NA|KX538968|MY_U464/12/Malaysia|2012_05_09 NA|KF923897|3269A/2012|China|2012_06 NA|KX538975|MY_U1024/12|Malaysia|2012_08_24 NA|KF923903/12691/2012|China|2012_05 NA|KX538977|MY_U41140/12Maysia|2012_05_02 NAIKX538969|MY U523/12|Malaysial2012-05-18 NA|KX538965|MY_U208/12|Malaysia|201203 28 NA|KX344031|OC43/human/Mex/LRTI_238/2011|Mexico|2011 0209 NA|KJ958218|LY341|China|2011_10_03 NA|KJ958219|LY342|China|2011_10_04 NA|KF923925|10574/2010|China|2010_09 NA|KF923922/8164/2009|China|2009_03 NA|KF923924/10290/2010|China|2010_07 NA|KF923918110108/2010 Khina1201005 NA|KF923893|2151A/2010|China|2010-07 NA|KF923923/892A/2008|China|2008 10 NA|KF923910|5445/2007|China|2007 06 NA|KF923914|5508/2007|China|2007 06 NA|KF923919|5595/2007|China|2007_07 NA|KF923908|5414/2007|China|2007_06 NA|KF923921/69A/2007|China|2007_05 NA|KF923907|5370/2007|China|2007_05 NA|KF923891/5240/2007/China|2007_05 NA|KF92391655192007|Chin|2007-06 NA|KF923911/5479/2007|China2007-06 NA|KF923913|5485/2007|China|2007 06 NA|KF923915|5517/2007|China|2007 06 NA|KF923920|5617/2007|China|2007_07 NA|KF923901/5472/2007|China|2007_06 NA|KF923917/5566/2007|China|2007_06 NA|KF923912|5484/2007|China|2007_06 NA|KF923892|5345/2007|China|2007_05 NA|KY554975|N09 382B|USA|2016 NA|KYG74920|N09-525B|USA|2016 NA|KY554974|N08_33B 360X|USA|2016 NA|KF923890|39A/2007|China|2007 04 NA|KY674918|N07_1647B|USA|2016 NA|KY554973|N07_1689B_116X|USA|2016 NA|KY554972|N07_1541B_433X|USA|2016 NA|KY674917|N07_1609B|USA|2016 D|JN129835|HK04_02|China|2004_11 NA|KF923899/3582/2006/China|2006_09 NAKF232305/220/2005|China2005 CIN129834/2204 $01 /$ China|2004 11

NAIKF530089|OC43/human/USA/911_66/1991|USA|1991 0129 NA|KF530082|OC43/human/USA/912_11/1991|USA|1991 02 NA|KF530094|OC43/human/USA/912_36/1991|USA|1991_02_22 NA|KF530079|OC43/human/USA/913_29/1991|USA|1991_03_14 NA|KF530076|OC43/human/USA/911_11/1991|USA|1991_01_03 NA|KF530071|OC43/human/USA/925_1/1992|USA|1992_05_04 NA|KF530091|OC43/human/USA/911_58/1991|USA|1991_01_24 NAIKF530096|OC43/human/USA/911_38/1991|USA|1991_01_23 NAIKF530095/OC43/human/USA/912 6/1991USA1991-01 05 NAIKF530067/OC43/human/USA/912 10/1991|USA|1991 02 - NA|KF530084|OC43/human/USA/951 18/1995|USA|1995 01 - NA|KF530098|OC43/human/USA/965 6/1996|USA|1996 $05-10$ NA|KF530068|OC43/human/USA/007_11/2000|USA|2000 07 27 NA|KF530081|OC43/human/USA/991_5/1999|USA|1999_01_07 NA|KF530070|OC43/human/USA/991_19/1999|USA|1999_01_15 NA|KF530063|OC43/human/USA/9612_48/1996|USA|1996_12_30 NA|KF530099|OC43/human//USA/971_5/1997|USA|1997_01_02 NA|KF30069|OC43/human/USA/982_4/1998/USA|1998_02_05 NA|MN306043|HCoV_OC43/Seattle/USAISC0841/2019|USA|2019 NA|KF923886|1908A/2010|China|2010_03 NA|KF923887/1997A/2010|China|2010 04 NA|KF923888|2145A/2010|China|2010_07 NA|KF923889|1926/2006|China|2006_03 NA|MF314143|HCoV_OC43/USAAACRI_0052/2016|USA|2016_03_07 NA|KY967360|HCoV_OC43/Seattle/USA/SC2476/2015|USA|2015 NA|KU131570|HCoV_OC43/UK/London/2011|United_Kingdom|2011_08_20 NA|KY014282|2007_09|France|2007 AA|KP198611/1783A10|China|2010_06 NAIKF923896/3074A/2012/China|2012 02 NA|KF923906/3194A/2012|China|2012 03 NA|KF530073|OC43/human/USA/8912_37/1989|USA|1989_12_21 NA|KF530061|OC43/human/USA/901_43/1990|USA|1990_01_19 NA|KF530066|OC43/human/USA901_33/1990|USA|1990_01_16 NA|KF530065/OC43/human/USA/901_41/1990|USA|1990_01_17 NA|KF530097|OC43/human/USA/9211_43/1992|USA|1992_11_30 NA|KF530074|OC43/human/USA/9212_33/1992/USA|1992_12_16 NAKKF53075/OC43/4m/USA1953 23/1995|USA11095-03-20 NAIKF530085/OC43/human/USA1871 25/1987|USA|1987 01 NA|KF530077|OC43/human/USA/873 16/1987|USA|1987 03 12 NA|KF530086|OC43/human/USA/872 5/1987|USA|1987-02 10 NA|KF530083|OC43/human/USA/873_19/1987|USA|1987 03 17 NA|KF530060|OC43/human/USA/851_15/1985|USA|1985_01_08 NA|KF530087|OC43/human/USA/873_6/1987|USA|1987_03_05 NA|KY014281|2002_04|France|2002 NA|KF530092|OC43/human/USA/008_5/2000|USA|2000_08_08 NA|KF530072|OC43/hman/USA/971__13/997/USA|1997_12_11 NAIKF530064OC43/human/USAI2_NAIKF530078|OC43/human/USA/9612_20/1996IUSA|1096 12 
